\title{
LA TESIS DEL YO EN LOS $\$$ 7-12 DE LA SEGUNDA INTRODUCCIÓN Y EN EL PRIMER \\ CAPÍTULO DEL ENSAYO DE UNA NUEVA EXPOSICIÓN DE LA DOCTRINA DE LA CIENCIA \\ DE FICHTE (GA I/4, 491-534)
}

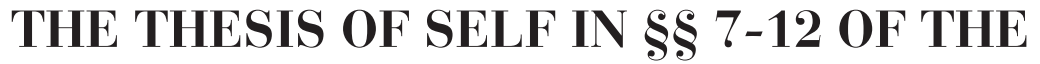 SECOND INTRODUCTION AND IN THE FIRST CHAPTER OF FICHTE'S AN ATTEMPT AT A NEW PRESENTATION OF THE DOCTRINE OF SCIENCE (GA I/4, 491-534)}

Gustavo Macedo Rodríguez

MLU-Halle

0 .

El estudio de la filosofía de Fichte da lugar, cuando se trata de interpretar y de analizar las principales tesis de su sistema filosófico, a un problema y a una exigencia. El problema consiste, por un lado, en que el estilo de la gran mayoría de sus textos es de difícil acceso para la interpretación del lector, generando muchas veces equívocos en la comprensión de los temas y problemas que Fichte intenta resolver o problematizar. La exigencia, por otro lado, adquiere sentido si se asume esta complicación, esto es, si la obra fichteana presenta un complejo sistema filosófico de argumentación, resulta una exigencia reconstruir cuidadosamente cada una de sus líneas argumentales. Dicho en otros términos, la comprensión y reconstrucción de la filosofía fichteana requiere no sólo del dominio de la jerga fichteana, sino, esencialmente, de la "traducción» de la misma. Este ultimo 
momento es posible en la medida en que sean problematizadas sus tesis de manera precisa. En el presente texto pretendo reconstruir una de las principales tesis fichteanas desde una perspectiva filosófica que apela, fundamentalmente, a la idea de clarificar su argumentación. Esto es, se trata aquí por un lado de analizar y clarificar argumentos presentados por Fichte y, por otro, de «traducirlos» a un lenguaje filosófico más accesible. Desde esta posición es posible cristalizar no sólo la reconstrucción y comprensión del sistema filosófico fichteano, sino también enriquecer el debate contemporáneo. Este análisis excluye claramente el contexto histórico de la obra, cuya importancia no será discutida aquí.

El presente texto tiene, pues, como objetivo principal tematizar una de las nociones fundamentales del sistema fichteano: el concepto del Yo. Éste será investigado específicamente en los $\S$ 7-12 de la Segunda Introducción $(Z E)$ y el primer capítulo del Ensayo de una nueva exposición de la Doctrina de la Ciencia (Versuch einer neuen Darstellung der Wissenschaftslehre) de Fichte. Este análisis permitirá en un segundo momento incursionar en algunas de las discusiones contemporáneas, especialmente en aquellas donde se niegan los alcances o posibilidades de dicho concepto dentro de una teoría de la subjetividad ${ }^{1}$. Antes de comenzar con la reconstrucción de la tesis del Yo en los ya mencionados apartados, resulta útil contextualizar el proyecto fichteano de manera general.

1.

La teoría de la subjetividad fichteana tiene como principal objetivo demostrar que un determinado acto de la conciencia, el cual describe un acto cuyo referente es él mismo, puede funcionar como fundamento o condición de posibilidad de la experiencia humana ${ }^{2}$. Dicho de otra manera, el proyecto fichteano consiste en explicar cómo y bajo qué condiciones es posible postular un

${ }^{1}$ Como ejemplos emblemáticos, podemos mencionar los planteamientos de Ernst Tugendhat o Peter Frederick Strawson. Véase E.Tugendhat, Selbstbewusstsein und Selbstbestimmung, Frankfurt am Main 1979; y P.F Strawson, Kant's Paralogismus: Self-Consciousness and the «Outside Observer», en Theorie der Subjektivität, comp. a cargo de Konrad Cramer, Frankfurt am Main 1990.

2 Esta Idea es expresada desde las primeras líneas de la Fundamentación de toda doctrina de la ciencia de 1794/95. Cf. GA, II, I, 91. 
principio absoluto del saber humano. Este programa teórico, llamado también en la Fundamentación a toda doctrina de la ciencia de 1794/95 «Historia pragmática del saber humano» ${ }^{3}$, es desarrollado por Fichte de formas diversas a lo largo de su obra. Esto es evidente si contrastamos la forma de argumentación del texto Fundamento de toda Doctrina de la Ciencia de 1794/95 (Grundlage der gesamten Wissenschaftslehre 1794/95) con la Doctrina de la ciencia nova methodo (Wissenschaftslehre nova methodo) o con el Intento de una nueva exposición de la Doctrina de la Ciencia (Versuch einer neuen Darstellung der Wissenschsftslehre $)^{4}$. En la GL, Fichte desarrolla una teoría basada en la explicación de un principio fundacional, cuyo comienzo teórico se caracteriza a partir de la proposición $A=A$, misma que sólo puede ser justificada bajo la presuposición de una actividad auto-productiva, a saber, la Tathandlung. ${ }^{5}$ Fichte intenta, de este modo, explicar y presentar de manera problemática tanto las contradicciones generadas por la actividad del Yo, así como las posibles disoluciones de las mismas (véase especialmente el $§ 5$ de la GL). La metodología explicativa de esta obra responde, podría decirse, a un proyecto basado esencialmente en deducciones trascendentales o genéticas. Por deducción o demostración genética Fichte entiende el examen de las condiciones de posibilidad de las funciones cognitivas del Yo, cuyo contenido yace en las propias leyes de la auto-posición del Yo. Esto implica, por un lado, que la deducción de las funciones cognitivas del sujeto dependen de la actividad auto-productiva del Yo (Tathandlung). Y por otro lado, que la teoría tanto de las funciones cognitivas del Yo como también de las acciones prácticas sólo puede ser fundada desde la perspectiva de la primera persona (desde una teoría trascendental à la Kant) y no desde el punto de vista de «alguna inteligencia externa al Yo», esto es, no desde una conciencia empírica. En contraste, el análisis metodológico tanto de la WLnm como del Versuch versan sobre la posibilidad de una deducción «externa», apoyada

${ }^{3}$ GA, II, I, 222

${ }^{4}$ En adelante las obras serán abreviadas de la siguiente manera: Grundlage de gesamten Wissenschaftslehre 1794/95= GL; Wissenschaftslehre nova methodo= Wlnm; y Versuch einer neuen Darstellung der Wissenschaftslehre= Versuch. Segunda introducción=ZE.

5 El concepto de la Tathandlung, es fundamental en la exposición del sistema fichteano. La función del concepto es describir un estado-acto de la conciencia desde el cual es posible la investigación de las funciones cognitivas del ser humano.

${ }^{6}$ Cabe mencionar que hay una relación de líneas argumentales entre estos dos textos, la cual se ha hecho evidente luego del descubrimiento de la obra Wissenschaftslehre nova methodo (Cf. GA I/4. 169- 181). 
fundamentalmente en la distinción entre la perspectiva de Yo y la perspectiva del filósofo. Este viraje en el método expositivo es corroborado por Fichte en dos cartas. ${ }^{6}$ Primero, en una carta dirigida a Reinhold en marzo de 1797, donde acepta la falta de "completitud [argumentativa] de la Grundlage [1794]»; Y en ese mismo mes, pero de 1799, en una carta dirigida a Johann Ernst Christian Schmidt. En ella, Fichte expresa que la insuficiencia de su anterior exposición [La exposición de la GL 1794/95] no radica en la falibilidad de sus principios, sino más bien en la deducción utilizada, dentro de la cual se suelen encontrar muchas insuficiencias.» ${ }^{8}$

Una primera aproximación a esta nueva estrategia emprendida por Fichte en WLnm y en Versuch puede ser caracterizada en términos generales de la siguiente manera: Fichte intenta fundamentar las condiciones de posibilidad de toda conciencia desde la estructura misma de la auto-producción o autoposición del Yo, acentuando, a diferencia de la GL, el carácter absoluto del Yo. ${ }^{9}$ En este sentido, Fichte acentuará la capacidad del Yo de auto-constituirse o auto-ponerse. Esta idea acentúa el carácter performativo del Yo, el cual, en términos contemporáneos, acentúa el rol de la primera persona en la adscripción de sus propios estados mentales.

En lo que sigue no abordaré más las posibles implicaciones de las correcciones o añadidos al interior de la obra fichteana. Más bien, y con un especial interés en el análisis del concepto del Yo, me interesa presentar una reconstrucción de la tesis del Yo planteada por Fichte, como se ha dicho, tanto en la $Z E \S 7$ - 12 como en el primer capítulo de Versuch. A partir de esta reconstrucción, pretendo mostrar las posibles dificultades y alcances teóricos de la misma.

7 Ibid.

8 Ibid.

9 Si bien es cierto que también en la $G l$ el análisis de la estructura del Yo intenta describir las condiciones de posibilidad de toda autoconciencia no debemos olvidar las inconsistencias argumentativas al interior de la $G L$. No debemos olvidar que Fichte se enfrenta a muchos problemas cuando en el $\S 4$ introduce el teorema del impulso inicial (Anstoß-theorem). Los problemas propios del tránsito de una estancia externa a un Yo absoluto parecen no estar presentes en sus obras posteriores, donde, como he mencionado arriba, se acentúa la explicación desde la estructura misma de la auto-producción del Yo. Véase Violetta Waibel. Hölderlin und Fichte. 1794-1800, Paderborn: München, 2000. 


\section{La tesis de la conciencia inmediata. EI Yo en «Versuch einer neuen Darstellung der Wissenschaftslehre. Lectura de los $§ 7$ - 12 de la ZE y del primer capítulo}

El texto Versuch fue publicado en partes, sin llegar a completarse, entre 1797 y 1798 en la revista «Philosophisches Journal einer Gesellschaft Teutscher Gelehrten», la cual Fichte editaba conjuntamente con Friedrich Immanuel Niethammer. Una de las tesis principales es demostrar que un acto de la conciencia, entendido éste básicamente como un acto auto-constitutivo del Yo, es decir, un acto no-intencional (unmittelbares Bewusstsein), puede postularse esencialmente intencional ${ }^{10}$, puede funcionar como un principio fundador del saber humano.

El argumento fichteano parte del supuesto de que todo juicio o contenido proposicional presupone un Yo, entendido éste como una función cognitiva y actuante que posibilita la adscripción de predicados. Esta condición debe ser expresada no como un «referente» general en un sentido psicológico, esto es, no como una conciencia inmediata de uno mismo desde la perspectiva de una conciencia empírica. Más aún, Fichte sostiene la idea de que el Yo es una condición necesaria para poder representar cualquier tipo de objeto. Dicho de otra forma, Fichte pretende demostrar que la conciencia inmediata de uno mismo es la condición de toda conciencia empírica. Esta conciencia inmediata es un acto de la conciencia (intellektuelle Anschauung). Aquí, conciencia inmediata refiere a un acto de la conciencia generado por la capacidad productiva del sujeto, cuya actividad describe un factum en la conciencia. En términos contemporáneos, Fichte se refiere a la capacidad cognitiva del Yo de producir objetos (actos mentales) y adscribirles predicados (P. ej. el de la infinitud). Esta capacidad describe un carácter no-intencional del $\mathrm{Yo}^{11}$. Con ello, es posible ver claramente que la noción del Yo en Fichte responde

${ }^{10}$ El término Intencionalidad del Yo no es un término utilizado por Fichte. En este ensayo refiere a la capacidad de auto-producirse o auto-constituirse.

${ }^{11}$ El concepto de la «intencionalidad» no es un término ficteano. No obstante, es posible establecer una similitud conceptual, bajo ciertas aclaraciones, entre el carácter intencional del Yo en Husserl y en Fichte. En Ideen Husserl caracteriza la intencionalidad como una estructura fundamental de la conciencia y de formas específicas de la relación de la conciencia con el mundo. Esta

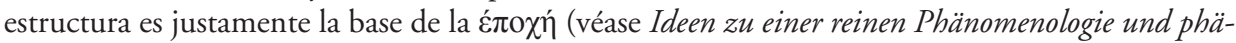
nomenologischen Philosophie, Husserliana, Gesammenlte Werke, Band III/1 \$ 30-34). Además, en el texto Cartesianische Meditationen escribe lo siguiente: «Die Vielfältigkeit der Intentionalität, die zu jedem cogito gehört, zu jedem weltlich bezogenen cogito schon dadurch, daß es nicht nur Welt- 
a una concepción «ampliada» del Yo trascendental kantiano. Para Fichte, el Yo no es sólo una condición de posibilidad de la experiencia (como sí lo es para Kant), sino más aún, él es el substrato de toda conciencia que es susceptible de representar objetos externos. Por ello, el Yo es una función no sólo cognitiva intencional, esto es, no solamente describe un acto de la conciencia reflexiva. Fichte caracteriza este acto como la condición de posibilidad de toda autoconciencia, es decir, como el presupuesto del carácter reflexivo del Yo. Dicho esto, surgen las siguientes preguntas: ¿¿A qué tipo de implicaciones nos conduce este presupuesto? Y ¿̨cómo debe ser entendida esta caracterización del Yo?

Primeramente es preciso aclarar que la noción fichteana de la "conciencia inmediata» debe ser distinguida de otras formas coloquiales de conciencia. No se le debe confundir ni con la noción de conciencia individual, ni mucho menos con la de conciencia personal. Estas últimas formas de conciencia remiten a estados de conciencia empírica. Cuando hablamos de una conciencia individual nos referimos a un sujeto que sabe que es idéntico al sujeto de la percepción, es decir, distingue entre sus representaciones del mundo externo y el actor de esas representaciones. Mientras que la conciencia personal se identifica con una forma de conciencia corporal, donde el sujeto no es capaz de identificarse numéricamente, sino sólo a partir de la descripción de sus características. ${ }^{12}$

Esta distinción es relevante por dos razones. Primeramente, porque ella prefigura una línea argumental del idealismo alemán que comienza a desmarcarse de la

liches bewußt hat, sondern selbst, als cogito, im inneren Zeitbewußtsein bewußt ist, ist nicht thematisch erschöpft in der bloßen Betrachtung der cogitata als aktueller Erlebnisse.»(Cartesianische Meditationen und pariser Vorträge.Husserliana, Gesammelte Werke. Band I, Haag, Martinus Nijhoff, 1973.Pág. 57). Puesto en esos términos, el carácter intencional del Yo en Husserl refiere a una función y estructura de la subjetividad similar a la capacidad de producción del Yo fichteano. Ese es justamente el sentido que queremos enfatizar con el termino intencionalidad del Yo. Es necesario, sin embargo, aclarar que Husserl se desmarca de la perspectiva idealista y sostiene que el Yo no es la función básica del sujeto y no puede, por ello, ser el fundamento de la filosofía. Otro referente importante en relación con el término de la intencionalidad es, dentro de la tradición analítica anglosajona, la filosofía de John Searle. Dicho rápidamente, la intencionalidad es para Searle una función biológica siempre relacionada con un objeto de la conciencia. Searle define intencionalidad del siguiente modo: «Intentionality is that property of many mental states and events by which they are directed at or about or of objects and states of affairs in the world.» (John Searle. Intentionality. An essay in the philosophy of mind. Cambridge university press, 1983, P. 1)

${ }^{12}$ Cf. Katja Crone, Fichtes Theorie konkreter Subjektivität. Untersuchung zur »Wissenschaftslehre nova methodo« (2005). 
perspectiva psicologista: se trata aquí de desarrollar la idea de un acto de la conciencia cuyo objeto de estudio no es un estado psicológico, es decir, no se trata de un determinado estado de conciencia donde son representados objetos de la experiencia empírica. Por el contrario, el punto de partida de la argumentación es una función que funda y que actúa (simultáneamente), posiblitando toda representación de la conciencia que posibilita toda representación. Dicho de manera breve, es necesario distinguir entre dos acepciones en las que se involucra el concepto del Yo, esto es, distinguir entre una conciencia empírica y una conciencia inmediata trascendental (intuición intelectual). Esta distinción es relevante, además, porque por medio de ella es posible distanciar la postura fichteana de cualquier imputación de psicologismo, ubicándonos más bien en los dominios y alcances de la perspectiva de la primera persona y su carácter intencional. Bajo este esquema, el proyecto fichteano genera formas argumentativas para responder a la pregunta: ¿̨bajo qué condiciones el Yo puede adscribirse predicados y «generar» estados de conciencia?

La respuesta a la anterior pregunta implica el análisis de la función cognitiva del Yo. Con ayuda de las distinciones hechas, es posible abordar de manera más precisa la tesis del Yo en los ya mencionados pasajes. Para Fichte la noción de conciencia inmediata y su caracterización dentro de la Doctrina de la ciencia es de especial interés. Fichte nombra esta conciencia inmediata de distintas formas a lo largo del texto: intuición de sí mismo, auto-posición, conciencia que retorna así misma o ipseidad. Como ya se ha mencionado, Fichte entiende por conciencia inmediata un acto sui generis de la conciencia que implica una actividad auto-constitutiva. Puesto que la posibilidad de dicho acto constituye la base teórica de la postulación de un principio fundamental, Fichte intenta mostrar la plausibilidad y las características de dicho acto. Esta argumentación consta de varios pasos. La tesis central consiste en mostrar que toda conciencia está condicionada por una conciencia inmediata. Esto es, que la condición de posibilidad de toda conciencia (empírica o intencional) es la conciencia inmediata de uno mismo. El punto de partida de la argumentación se vincula con la distinción metodológica entre el pensarse a uno mismo y pensar un objeto externo, d.i. la distinción entre una conciencia inmediata y una conciencia empírica o intencional. Dicho de otra manera, que es necesario tener conciencia inmediata de uno mismo, para sí, tener conciencia de los objetos externos. Si la condición para poder representar objetos del mundo externo es ser consciente de uno mismo como determinado en el tiempo, entonces toda representación presupone un estado de autoconciencia inmediato. 
Puesto en términos más concretos. Cuando pensamos una pared o una mesa, el estado de conciencia remite a una función mediada. En cambio, pensarse así mismo no requiere mediación. Esta distinción de modos o estados de autoconciencia es posible porque que hay una distinción básica en todo juicio: el que piensa y lo que es pensado. Como mera posibilidad el concepto del Yo puede ser objeto del pensar. Para demostrar la viabilidad de este acto inmediato Fichte apela al supuesto de que toda representación, sea de un objeto o de uno mismo, implica ya una actividad. Tanto pensar una pared como pensarse a sí mismo son actividades cognitivas del Yo. En ellas, es posible distinguir que cuando pienso un objeto hay dos elementos contenidos en el juicio, esto es, el que piensa y lo pensado. En ese sentido, el concepto del Yo puede definirse de la siguiente forma: El concepto del Yo o el Yo entendido intencionalmente describe el actuar del Yo sobre sí mismo. De ahí que Fichte afirme que el Yo es una función cognitiva que esencialmente describe una actividad. Fichte expresa esta idea del siguiente modo: «El concepto o el pensar del Yo consiste en la acción misma del Yo, e inversamente, esa misma acción genera necesariamente un pensar del Yo, y ningún otro.» ${ }^{13}$ Si nos preguntamos por la condición trascendental de dicha actividad, la respuesta sería: toda actividad de la conciencia, incluida la de la conciencia inmediata de uno mismo, implica la predicación, es decir, se aplica un concepto (I, 532). Ya en la GL, Fichte adscribe a la noción de actividad infinita del Yo, concepto decisivo en la explicación del teorema del Anstoß y, por añadidura, en la demostración genética del § 5, dos aspectos dentro de la exposición de su sistema: por un lado la actividad del Yo muestra su carácter predicativo, esto es, la capacidad para adscribir un predicado y por ello limitar la función del Yo. Y, por otro, la capacidad de producir o adscribir una actividad infinita del Yo, es decir, la aceptación del carácter intencional de la conciencia. ${ }^{14}$ En ese mismo sentido, es posible caracterizar el primer momento del concepto del Yo como la aplicación de un concepto en el acto mismo del pensar o actuar, cuyo objeto es él mismo.

El resultado de la anterior reconstrucción es la aceptación de la posibilidad de podernos pensar a nosotros mismos como objetos de nuestra conciencia. Justo en esta afirmación es posible ver el distanciamiento fichteano de la filosofía trascen-

${ }^{13}$ En el texto original: «Der Begriff oder das Denken des Ich besteht in dem auf sich Handeln des Ich selbst; und umgekehrt, ein solches Handeln auf sich selbst giebt ein Denken des Ich, und schlechthin kein anderes Denken.»GA, I/4, 523.

14 Para una mayor comprensión de la actividad infinita del Yo, véanse los $§ \S 4$ y 5 de la GL. 
dental de Kant. Pues es justo la postulación de un objeto de conocimiento de la conciencia que es esencialmente intencional y que refiere a sí mismo una de las tesis negadas por el idealismo trascendental kantiano. Para Kant, no es posible deducir conocimiento alguno de la mera representación del Yo. Éste debe necesariamente referir a una representación objetiva, si se pretende hablar de conocimiento y experiencia en sentido estricto. Es decir, la representación del Yo no produce por sí mismo conocimiento alguno.

Bajo esta perspectiva podríamos afirmar que el Yo entendido como conciencia inmediata, tiene hasta ahora dos funciones, por un lado es condición de posibilidad de objetos externos a la conciencia y por otro, es condición de toda conciencia. La deducción de una actividad del Yo cuyo objeto es él mismo es posible sólo bajo el presupuesto de que el pensar y el actuar de una conciencia son idénticos. Bajo esta perspectiva el Yo no es más que una aplicación de su propio concepto cuando se piensa. Esta acción describe exactamente lo que sucede cuando se aplica algún predicado. Por ello, una intuición de uno mismo implica un acto de la espontaneidad o de la libertad. El Yo es el desarrollo conceptual de la autoposición de uno mismo como objeto, como actividad. Para mostrar la plausibilidad de esta tesis, Fichte distingue entre una conciencia general, la cual se realiza bajo la mera aplicación de conceptos, y una conciencia de la intuición, la cual tiene lugar a partir de su propio actuar, a saber, a partir de una auto-producción. El conceptualismo o inferencialismo explícito de Fichte converge con la idea de un acto de la conciencia constitutivo que da lugar a la aclaración de la conciencia empírica. Aquí es posible plantear la pregunta: ¿quiere decir esto que la noción del Yo no tiene un estatus privilegiado en su Programa teórico? Implícitamente y a la luz de comparaciones conceptuales con la Wlnm y la $G L$, es posible visualizar que su tesis del Yo como principio de todo saber tiene dos aspectos que lo constituyen: El Yo es una función cognitiva-actuante construida a partir de la libertad humana, esto es, es un resultado de la espontaneidad; pero también, es el resultado de un acto de la reflexión, concebido a partir de la aplicación de un concepto. Esto es extensamente analizado por Fichte en los $\$ \$ 4$ y 5 de la GL. Allí Fichte explica la función de conceptos como infinitud o actividad infinita del Yo. Lo que no es lo suficientemente claro, sin embargo, es en qué sentido un acto auto-productivo puede ser condición de toda conciencia?

Autores contemporáneos como Peter Frederick Strawson y Ernst Tugendhat han argumentado que la función del Yo debe limitarse a una función cognitiva que 
debe ser entendida como una condición de posibilidad y no como una función capaz de funcionar como fundamento de toda experiencia posible. ${ }^{15}$ La principal crítica esgrimida por estos autores, grosso modo, es que un acto de la conciencia no puede per se producir conocimiento. El resultado de esta representación del Yo en tanto objeto, es decir, fuera del contexto de su relación trascendental con el mundo externo, no puede producir contenidos con validez objetiva, ya que no tiene referente empírico y sólo es condición de posibilidad del representar en general. La función del Yo queda limitada bajo este esquema a una mera condición de posibilidad del conocimiento.

Luego de haber analizado la tesis central del Yo en los apartados arriba mencionados, es posible rechazar esta crítica. La noción del Yo en Fichte no refiere, como lo hemos visto, a algún conocimiento empírico o se reduce a una condición de posibilidad. A Fichte le interesa sobremanera mostrar que el Yo contiene un carácter intencional que no refiere al mundo fenoménico, pero que está en constante interacción con él, esto es, que hace posible la representación del mundo fenoménico. La justificación de este carácter intencional del Yo tiene lugar dado el carácter orereflexivo o no intencional su existencia, esto es, debido a la conciencia inmediata de su actuar. De esta maenera queda claro que la concepción fichteana del Yo responde a un proyecto performativo y auto-constitutivo. El objetivo principal de Fichte es demostrar que hay un carácter intencional en la estructura del Yo y que en ese sentido no puede reducirse esta estructura al mundo fenoménico. Con ello, podemos concluir que la noción del Yo en Fichte debe ser entendida como una compleja estructura que no puede ser limitada al mundo empírico. Queda por mostrar si esa estructura puede realmente ser el fundamento de todo el saber humano. Esta tarea programática debe ser reconstruida en otra ocasión.

15 Véase particularmente: Ernst Tugendhat, Selbstbewusstsein und Selbstbestimmung, Frankfurt am Main 1979; y P.F Strawson, Kant's Paralogismus: Self-Consciousness and the "Outside Observer», en Theorie der Subjektivität, Konrad Cramer et.al, Frankfurt am Main 1990. 